\title{
CARACTERÍSTICAS Y EVOLUCIÓN DE LA OFERTA DE ALOJAMIENTO DE AIRBNB EN UNA CIUDAD PATRI- MONIO CULTURAL DE LA HUMANIDAD. CASO CUEN- CA (ECUADOR)
}

Characteristics and evolution of the Airbnb accommodation offer in a cultural heritage city of humanity. Case Cuenca (Ecuador)

Freddy Edgar Espinoza Figueroa

Contactos: freddy.espinoza@ucuenca.edu.ec

Segundo Amador Freire Chaglla segundo.freire@ucuenca.edu.ec

Proyecto de análisis de indicadores de sostenibilidad y competitividad turística con incidencia en las estrategias de gestión sostenible, gobernanza y políticas públicas del cantón Cuenca, Departamento de Investigación de la Facultad de Ciencias de la Hospitalidad, Universidad de Cuenca 


\section{Resumen}

Actualmente han surgido modelos de negocio innovadores en cuanto a su operación, a través de medios de gestión electrónica, como Airbnb (alojamiento), están presentes casi en todo el mundo, bajo la denominación de economía colaborativa, tienen predilección por ubicarse en asentamientos urbanos consolidados como centros históricos; Cuenca, Patrimonio Cultural de la Humanidad no es la excepción. No obstante, generan incomodidad a los modelos de negocio tradicionales y menoscaban la autenticidad, integridad y valores patrimoniales de los bienes culturales edificados en virtud de una probable sobreoferta de alojamiento. Este estudio de tipo descriptivo se realizó mediante la recopilación de datos históricos (2010 - 2017) de la oferta de Airbnb (página web) para Cuenca, y, tiene como objetivo, poner en contexto las características y evolución de este tipo de oferta en un contexto de ciudad patrimonial, teniendo como resultado una tendencia a la concentración de este tipo de establecimientos en áreas históricas y patrimoniales; además se evidencia una tendencia decreciente de las tarifas frente a una presión a la reducción de los precios de los establecimientos regulados. Este fenómeno tiene efectos más allá del turismo, por lo que es alarmante la falta de políticas de control y regulación por parte del sector público, la desidia en torno a la innovación por parte del sector privado, la falta de estudios y el menoscabo del patrimonio cultural edificado y la vivienda en relación al incremento de una oferta informal.

Palabras clave: Centro histórico, Patrimonio Cultural, Airbnb, alojamiento turístico, Cuenca (Ecuador)

\section{ABSTRACT}

Currently, innovative business models have emerged in terms of operation, through electronic management means, such as Airbnb (accommodation), are present almost worldwide, under the name of collaborative economy, have a predilection for locating in urban settlements consolidated as historical centers; Cuenca, Cultural Patrimony of the Humanity is not the exception. However, they generate discomfort to traditional business models and undermine the authenticity, integrity and heritage values of cultural assets built by virtue of a probable oversupply of accommodation. This descriptive study was conducted through the collection of historical data (2010 - 2017) of the Airbnb offer (web page) for Cuenca, and, aims to put in context the characteristics and evolution of this type of offer in a patrimonial city context, resulting in a trend towards the concentration of this type of establishments in historical and heritage areas; In addition, there is evidence of a downward trend in tariffs in the face of pressure to reduce the prices of regulated establishments. This phenomenon has effects beyond tourism, so it is alarming the lack of policies of control and regulation by the public sector, laziness around innovation by the private sector, the lack of studies and the ero- 
sion of heritage built culture and housing in relation to the increase of an informal offer.

Key words: Historical Center, Cultural Heritage, Airbnb, Tourist accommodation, Cuenca (Ecuador)

\section{Introducción}

El turismo es un fenómeno moderno con efectos visibles: en la economía, el entorno natural, las zonas edificadas, la población local de los territorios visitados y en los mismos visitantes (Cioce-Sampaio \& Silva-Segovia, 2015; Organización Mundial de Turismo (OMT), 2010).

A nivel mundial, actualmente existe un acalorado debate en virtud del surgimiento de un nuevo fenómeno de comercialización en respuesta a las crisis económicas y a los avances tecnológicos, llamados por algunos autores como economía colaborativa o compartida (Bostman \& Rogers, 2011; Bulchand Gidumal \& Melián Gonzáles, 2016; Kostakis \& Bauwens, 2014; Owyang, Tran, \& Silva, 2013; Pascual, 2015), economía del bien común (Felber, 2012), economía peer to peer (Pascual, 2015).

Sin embargo, aún faltan estudios en torno a estos temas, considerando que también se puede discutir y relacionar a este fenómeno con la economía solidaria $^{1}$ y economía popular ${ }^{2}$ (Coraggio, 2011), no obstante, al existir un intercambio de bienes y servicios con un medio de cambio (dinero), se denominaría economía capitalista de mercado (Martin, 2016).

En principio, estos modelos de negocio son distintos a los tradicionales en la forma en que se ejecutan: no compran nada, y no producen bienes y servicios; es decir, no siguen los modelos económicos clásicos (Bulchand Gidumal \& Melián Gonzáles, 2016). En teoría se basan en compartir y cooperar -sin olvidar el dinero- esto ha generado un rediseño en la oferta de bienes y servicios como el transporte, alojamiento, restauración, intermediación, entre otros, para lo cual ha sido trascendental la incursión de la tecnología como facilitador para obtener bienes y servicios (Pascual, 2015), es decir, una plataforma digital de intermediación que conecta a clientes y proveedores (Evans \& Schmalensee, 2016),

\footnotetext{
${ }^{1}$ La economía solidaria tiene que ver con la asociación y aglutinamiento de voluntades y dinero en igualdad de condiciones

${ }^{2}$ La economía popular es una economía familiar sin acumulación ampliada, es decir sin superávit, lo que busca es trabajo y que el mismo le permita satisfacer sus necesidades básicas hay intercambio de dinero.
} 
posibilitando reducir costes de transacción y aumentando la escala geográfica en las que se desarrollan (Pascual, 2015).

En las grandes ciudades del mundo, este fenómeno ha sido acusado de generar efectos como gentrificación (Deustche \& Gendel Ryan, 2015), turistificación y desertificación de zonas urbanas (Smith \& Williams, 2013), y, en el caso de centros históricos incluidos en la lista de Patrimonio Mundial han empezado a incumplir la premisa básica de la Convención del Patrimonio Mundial -cultural y natural- que hace alusión a la conservación y valoración del valor universal excepcional, aquello se evidencia en la región en casos como Machi Picchu y Galápagos en donde el incremento de instalaciones turísticas -como hoteles, restaurantes, entre otros- han ido en desmedro de la conservación de los valores por los que fueron incluidos en la Lista; es indispensable que los criterios de desarrollo adopten posturas técnicas (Caraballo Perichi, 2011).

El número de instalaciones turísticas en centros históricos puede resultar un gran problema considerando que muchas edificaciones son puestas como recurso económico (alojamiento) (Fernández de Paz, 2006). Hay un gran número de prestadores de servicios que inciden en esta nueva modalidad que, en la mayoría de casos, incurren en la competencia desleal (Bulchand Gidumal \& Melián Gonzáles, 2016), es decir, son agentes perturbadores de las estructuras de negocio ya establecidas a nivel social, económico y técnico (Martin, 2016).

Entre los casos más conocidos de plataformas de intermediación tenemos: Airbnb (alojamiento), Uber (transporte urbano), Get Around (vehículos), Parqex (garaje), Handy (tareas manuales), Trip4Real (experiencias para turistas), Nidmi (asistencia para familias), Etsy (productos artesanales), (Bulchand Gidumal \& Melián Gonzáles, 2016), entre otros.

Este tipo de servicios, en distintos ámbitos, compiten entre sí generando un modelo de crecimiento para ser la única empresa disponible, es decir, una máxima economía de escala posible (Bulchand Gidumal \& Melián Gonzáles, 2016). Mientras más grande es la plataforma en relación al mayor número de transacciones, los costos marginales de operación son menores, produciendo un efecto de interés por las mismas, tanto para oferentes como para demandantes; además, a mayor número de transacciones, mayor es la capacidad de previsión de lo que ocurre y ocurrirá en pro de una mejor gestión de recursos para la satisfacción de los usuarios (Bulchand Gidumal \& Melián Gonzáles, 2016), mediante el estudio del comportamiento de los destinos y usuarios (Ascanio Guevara, 2012).

La innovación y las alianzas estratégicas son fundamentales para estos negocios, tal es el caso de Uber (transporte privado), que dispone de un trato con 
una compañía de imágenes satelitales para optimizar la recogida de pasajeros, además, de servicios complementarios basados en la innovación como el lanzamiento de UberCENTRAL, para que las empresas reserven y paguen el transporte de sus clientes, y las pruebas realizadas en coches autónomos -sin pilotos- con la intención de reemplazar al conductor (Bulchand Gidumal \& Melián Gonzáles, 2016).

Las ciudades patrimoniales como Cuenca, no son excepciones en esa tendencia de irrupción comercial altamente concentrada en espacios sensibles por sus valores patrimoniales. Estos negocios, sobretodo de alojamiento con precios relativamente bajos, implican adaptar (no restaurar, ni rehabilitar) edificaciones para hospedaje con una oferta de servicios fuera del contexto legal, aquellas formas de comercio turístico conviven con formas legales de servicios de hospedaje, incrementando las instalaciones turísticas, provocando alta concentración en un espacio declarado Patrimonio Cultural de la Humanidad. Es así cómo, mediante esta investigación, deseamos poner en contexto las características y evolución que ha tenido la oferta de alojamiento de Airbnb en una ciudad que ostenta el título de Patrimonio Cultural de la Humanidad, como es el caso de Cuenca.

\section{El modelo de negocio de Airbnb}

Airbnb, es una plataforma de alojamiento, nacida en San Francisco, en agosto de 2008, a partir de dos situaciones: sus fundadores, Nathan Blecharcyk, Brian Chesky y Joe Gebbia, tenían una tarifa de alquiler por su residencia, que no podían costear, no obstante, y de manera fortuita para ellos, se dio una convención de diseñadores que saturó los alojamientos en su ciudad de residencia (San Francisco, EE. UU.). Es así como entre Nathan, Brian y Joe nace la idea de implementar en su vivienda camas inflables y, con el hospedaje brindar el desayuno. Esta idea tuvo un buen resultado en cuanto a la demanda y las ganancias generadas.

Luego de esta experiencia, los tres estadounidenses establecieron un sitio web que posibilita a las personas poder ofertar sus residencias como alojamiento turístico a cambio de dinero. Esta modalidad ha revolucionado la industria del alojamiento en aproximadamente una década.

Airbnb tiene dos tipos de clientes: anfitriones, que pueden poner a disposición sus instalaciones; y huéspedes, que requieren instalaciones de alojamiento. Estos actores crean una cuenta de usuario para interactuar sí, rigiéndose bajo 
las políticas de la empresa. En cuanto a la fijación de precios, las plataformas pueden intervenir o no, no obstante, Airbnb orienta al anfitrión sobre los precios a partir de sus datos históricos (evolución de la demanda) y de las características de la vivienda a través de una asistencia desinteresada; por otro lado, existe una asistencia con costo adicional (Smart Pricing), en donde la plataforma usa a su favor los datos históricos recopilados para generar ganancias. Adicionalmente mediante sistemas externos a la plataforma, como por ejemplo Beyond Princing, asisten a los proveedores de servicios y se encargan de gestionar de manera automática los precios en virtud de la demanda, estacionalidad, ubicación geográfica, entre otros aspectos, cobrando un $1 \%$ del total de ingresos de la propiedad (Bulchand Gidumal \& Melián Gonzáles, 2016).

Regularmente, el usuario cancela los valores por servicios directamente a la plataforma, luego esta se encarga de cancelar al proveedor del servicio (distancia temporal para prevenir el fraude); la comisión de servicios es tanto para el ofertante (entre 6 al 12\%) como para el demandante (3\%) (Bulchand Gidumal \& Melián Gonzáles, 2016; Vaughan \& Daverio, 2016). Una manera de indicador cualitativo son las evaluaciones que mutuamente se realizan entre huésped y anfitrión, esta valoración se tomará en cuenta para futuras transacciones.

En todo el mundo se ha generado un gran debate en torno a la normativa de regulación y tributación, considerando que muchas de estas actividades compiten directamente o se complementan a negocios similares regulados por una ley (Bulchand Gidumal \& Melián Gonzáles, 2016; Guttentag, 2015), dando paso en algunos sitios a acuerdos entre la plataforma y las administraciones públicas de las ciudades.

En Ámsterdam, por ejemplo, la plataforma se comprometió a recaudar la tasa turística (impuestos) de la ciudad (2015); en Francia se aprobó la "tasa fiscal Google" con el objetivo de sancionar las medidas de "optimización fiscal", práctica recurrente de las grandes multinacionales para evadir impuestos (Rojano Uscanga, 2016). Latinoamérica es uno de los mercados potenciales según la revista FORBES; en México se registra un crecimiento del $188 \%$ de usuarios (López, 2016); en Argentina se reporta un avance de Airbnb como opción de alojamiento (Pizarro, 2017).

El denominador común de esta irrupción es la incomodidad de las administraciones públicas y los empresarios privados (regulados), puesto que los prestadores de este servicio no cumplen con requisitos legales, ni de tributación, generando un escenario de competencia desleal condicionado por la irregularidad; sin embargo, existe un incipiente olvido en cuanto a efectos que generan en 
Freddy Edgar Espinoza Figueroa, Segundo Amador Freire Chaglla

Características y evolución de la oferta de alojamiento de Airbnb en una

ciudad patrimonio cultural de la humanidad. Caso Cuenca (Ecuador)

los espacios urbanos como áreas históricas y patrimoniales declaradas como Patrimonio Cultural de la Humanidad, que tienen como misión conservar su valor universal excepcional.

\section{Caso de estudio, Cuenca (Ecuador)}

En el caso del Ecuador (1.544.463 de llegadas en 2015; 1.418 .159 de llegadas en 2016; 750.101 de llegadas desde enero a junio de 2017) (Ministerio de Turismo, 2017) este nuevo modelo de negocio ha irrumpido en las ciudades más grandes del país. Recientemente, en Guayaquil, se prohibió Uber (Gonzáles, 2017). El escenario nacional turístico, en la actualidad, está caracterizado por la desactualización y ausencia de políticas, la desarticulación entre el sector público y privado, la ausencia de estadísticas confiables, y la promoción que no parte desde una estrategia de planificación, situaciones que, sumadas a otros aspectos propios de cada ciudad, han coadyuvado para que exista incomodidad.

En Cuenca, ciudad intermedia, con 600.000 habitantes aproximadamente, Patrimonio Cultural de la Humanidad ${ }^{3}$ (1999), actualmente sufre una grave recesión en relación al alojamiento, prueba de aquello son las cifras oficiales de ocupación (32\% en 2016) que exhibe la Fundación Municipal Turismo para Cuenca a través del Grupo de Investigación Económica Regional (GIER), la parte más afectada es quizá el Centro Histórico (área declarada patrimonio cultural de la humanidad $-2.24 \mathrm{~km}^{2}$ ) que alberga 133 establecimientos de alojamiento regulados, de un total de 223.

En la prensa se puede ver todo tipo de artículos que alegan dicha recesión: "La hotelería informal gana espacio" (El Mercurio, 2014), "La Asociación Hotelera de Cuenca se declara en crisis" (Expreso, 2016), "La competencia informal preocupa a los hoteleros", "El alojamiento informal es una realidad latente" (El Tiempo, 2016), "Operativos contra hoteles y restaurantes informales se inician en Cuenca" (Astudillo, 2016).

Es importante mencionar que la crisis del sector turístico también se relaciona con otros problemas como el aeropuerto, la construcción del Tranvía Cuatro Ríos -que atraviesa el Centro Histórico-, el interés de los extranjeros (en su mayoría jubilados estadounidenses y europeos) por Cuenca, no es exclusivamente por turismo, sino que acuden a la ciudad como un lugar para radicarse (García Álvarez, Osorio Guerrero, \& Pastor Herrera, 2017). Sin embargo, el problema más repetitivo es la informalidad en el alojamiento, la cual se ha vuelto

${ }^{3}$ Criterios UNESCO ii, iii, v 
queja común del sector privado. Con este punto de partida es necesario preguntarse: ¿Y el espacio? ¿Y los valores del Centro Histórico?

En el año 2001, las competencias de turismo fueron transferidas desde el Estado central hacia la Municipalidad de Cuenca, en el mismo año “...el Concejo Cantonal de Cuenca, constituyó y reguló el Consejo de Turismo del Cantón Cuenca, como el órgano descentralizado de Gobierno Local con competencia en la formulación de políticas y normas, la planificación, el control de establecimientos, el financiamiento y operación de la actividad turística cantonal, en el marco de las políticas nacionales y locales emitidas y sobre la base del Convenio de Transferencia de competencias entre el Ministerio de Turismo y la Municipalidad de Cuenca." (Ilustre Consejo Cantonal de Cuenca, 2005, párr. 6); posteriormente, en el año 2005, se deroga la ordenanza que regula el Consejo de Turismo del cantón Cuenca y se procede a conformar una persona jurídica de derecho privado -Fundación Municipal Turismo para Cuenca- que asume solamente la competencia de promoción turística (llustre Concejo Cantonal, 2005) labor que ha sido llevada a cabo aproximadamente por 12 años. Las tareas de control y regulación que solicitan los actores privados están repartidas entre la Dirección de Áreas Históricas y Patrimoniales (Centro Histórico) y Control Urbano (Área urbana y rural).

Cabe indicar que, en marzo del año 2015, se generó un reglamento de alojamiento turístico a nivel del país: Hotel ( 2 a 5 estrellas), hostal ( 1 a 3 estrellas), hostería ( 3 a 5 estrellas), hacienda turística ( 3 a 5 estrellas), lodge ( 3 a 5 estrellas), resort (4 estrellas a 5 estrellas), refugio (categoría única), campamento turístico (categoría única) y casa de huéspedes (categoría única); cada uno con requisitos en relación con su infraestructura, ubicación geográfica, calidad y servicios que prestan (Ministerio de Turismo, 2015). En Cuenca, actualmente, existen 223 establecimientos de alojamiento registrados en el Ministerio de Turismo, con 8.546 plazas, dividiéndose por clasificación en: hoteles $38 \%$, hostales $45 \%$, casa de huéspedes 9\%, y otros 8\% (Departamento de Investigación Facultad de Ciencias de la Hospitalidad, 2016).

El modelo de negocio denominado Airbnb ha irrumpido en la ciudad con 178 establecimientos registrados en su página web, los cuales cuentan con 581 plazas $(6.79 \%$ de plazas en relación a las plazas reguladas).

Es difícil aseverar que la recesión en el alojamiento turístico se debe exclusivamente a la irrupción de esta plataforma de intermediación en Cuenca, y también se torna complicado generar medidas como se ha hecho en otros países, como prohibiciones, aplicación estricta de la legislación, desarrollo de una 
legislación específica, imposición de más reglas, simplificación de la normativa (Bulchand Gidumal \& Melián Gonzáles, 2016) o tomar cualquier decisión sin saber qué sucede en el espacio, es decir, los fenómenos sociales, económicos, culturales, entre otros, que suceden en el Centro Histórico de Cuenca considerando que "(...) el Centro Histórico de la ciudad de Cuenca constituye testimonio trascendental de la Cultura Ecuatoriana (...) Patrimonio Cultural de la Humanidad, por cumplir con los criterios 2, 4 y 5 de la Convención de Patrimonio Mundial (...) cuna de varias culturas, cuyas expresiones se deben preservar, conservar y difundir" (Ilustre Consejo Municipal de Cuenca, 2010, p. 1) por lo tanto se "...conservará sin alteraciones las características funcionales, formales y constructivas, en todas las edificaciones inventariadas con grado de valor patrimonial, de acuerdo a su categorización. Se mantendrá y consolidará los elementos distributivos tales como: patios, galerías, jardines, corredores, huertos, etc., y de igual manera sus detalles constructivos y decorativos de valor" (Ilustre Consejo Municipal de Cuenca, 2010, p. 13).

\section{Metodología}

El estudio tuvo un enfoque descriptivo con el fin de conocer la oferta de Airbnb en Cuenca y su comportamiento mediante la evaluación estadística de datos históricos. Se realizó un catastro de la oferta de Airbnb en Cuenca usando el software Excel, tomando datos expuestos en su página web como: la capacidad, el precio, las evaluaciones (comentarios), la ubicación y el año de registro.

De los datos recogidos se realizaron cuatro análisis mediante el software de visualización de datos interactivos Ilamado Tableau Public. El primer análisis buscó establecer la relación entre la capacidad (plazas), el tipo de hospedaje (tal cual se oferta en la web) y la ubicación geográfica no específica (dentro y fuera del Centro Histórico) ${ }^{4}$ (Gráfico 1).

El segundo análisis expuso, mediante datos históricos, la relación entre la suma acumulada de capacidad ofertada y la media móvil de precio ofertado (Gráfico 2).

El tercer análisis buscó, mediante datos históricos, la relación entre el promedio de plazas ofertadas y el promedio de tarifas aplicadas y se construyó un indicador que midió el valor en dólares de la tarifa por persona como precio/plaza que estima el movimiento de la tarifa en el tiempo (Gráfico 3).

\footnotetext{
${ }^{4}$ Para saber la ubicación geográfica exacta se necesita hacer la reserva mediante tarjeta de crédito, puesto que, al revisar las ofertas, solamente aparece un polígono en el que puede estar situada la propiedad; se ha optado por clasificarlo en dos: dentro y fuera del Centro Histórico.
} 
Freddy Edgar Espinoza Figueroa, Segundo Amador Freire Chaglla

Características y evolución de la oferta de alojamiento de Airbnb en una

ciudad patrimonio cultural de la humanidad. Caso Cuenca (Ecuador)

Finalmente se analizó, mediante datos históricos, el promedio de evaluaciones (comentarios) de usuarios de servicios de Airbnb (Gráfico 4).

\section{Resultados}

Los datos obtenidos de la página de Airbnb han sirvieron como elemento para caracterizar la oferta de este tipo de alojamientos en Cuenca, arrojando una serie de datos que han sido procesados para tener una mayor perspectiva de lo que sucede en la ciudad. A continuación se exponen cuatro análisis detallados en la parte metodológica del estudio.

\section{Gráfico 1}

Relación entre la capacidad (plazas), tipo de hospedaje y ubicación geográfica no específica

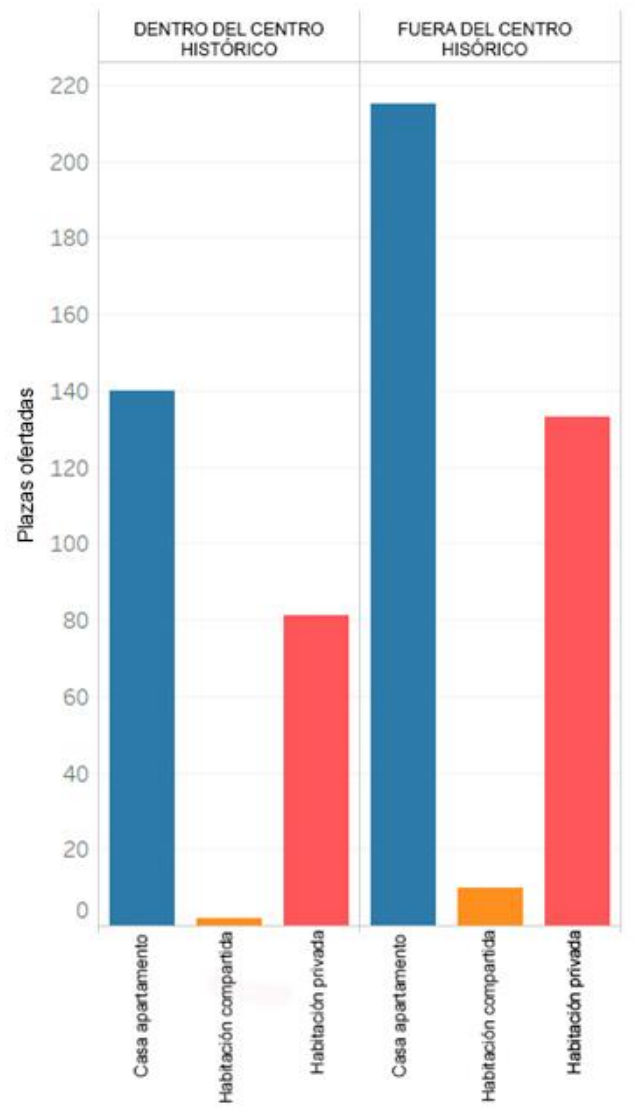

El gráfico 1 demuestra que dentro del Centro Histórico están instaladas 223 plazas, de las cuales 140 pertenecen a casas apartamento, 2 representan a 


\section{Freddy Edgar Espinoza Figueroa, Segundo Amador Freire Chaglla}

Características y evolución de la oferta de alojamiento de Airbnb en una

ciudad patrimonio cultural de la humanidad. Caso Cuenca (Ecuador)

habitaciones compartidas y 81 a habitaciones privadas. Fuera del Centro Histórico (zona urbana y rural) (INEC, 2015) existen 358 plazas, de las cuales 215 pertenecen a casas apartamento, 10 a habitaciones compartidas y 113 a habitaciones privadas.

Estos datos demuestran que el Centro Histórico es el espacio predilecto para este tipo de hospedajes, sobre todo de casas apartamento y habitaciones privadas y, que fuera del Centro Histórico, existe una mayor cantidad en cuanto a plazas que en su mayoría resultan ser casas apartamento y habitaciones privadas, sin embargo, el espacio es absolutamente mayor a la porción declarada Patrimonio Cultural de la Humanidad.

Gráfico 2

Histórico de oferta - precio

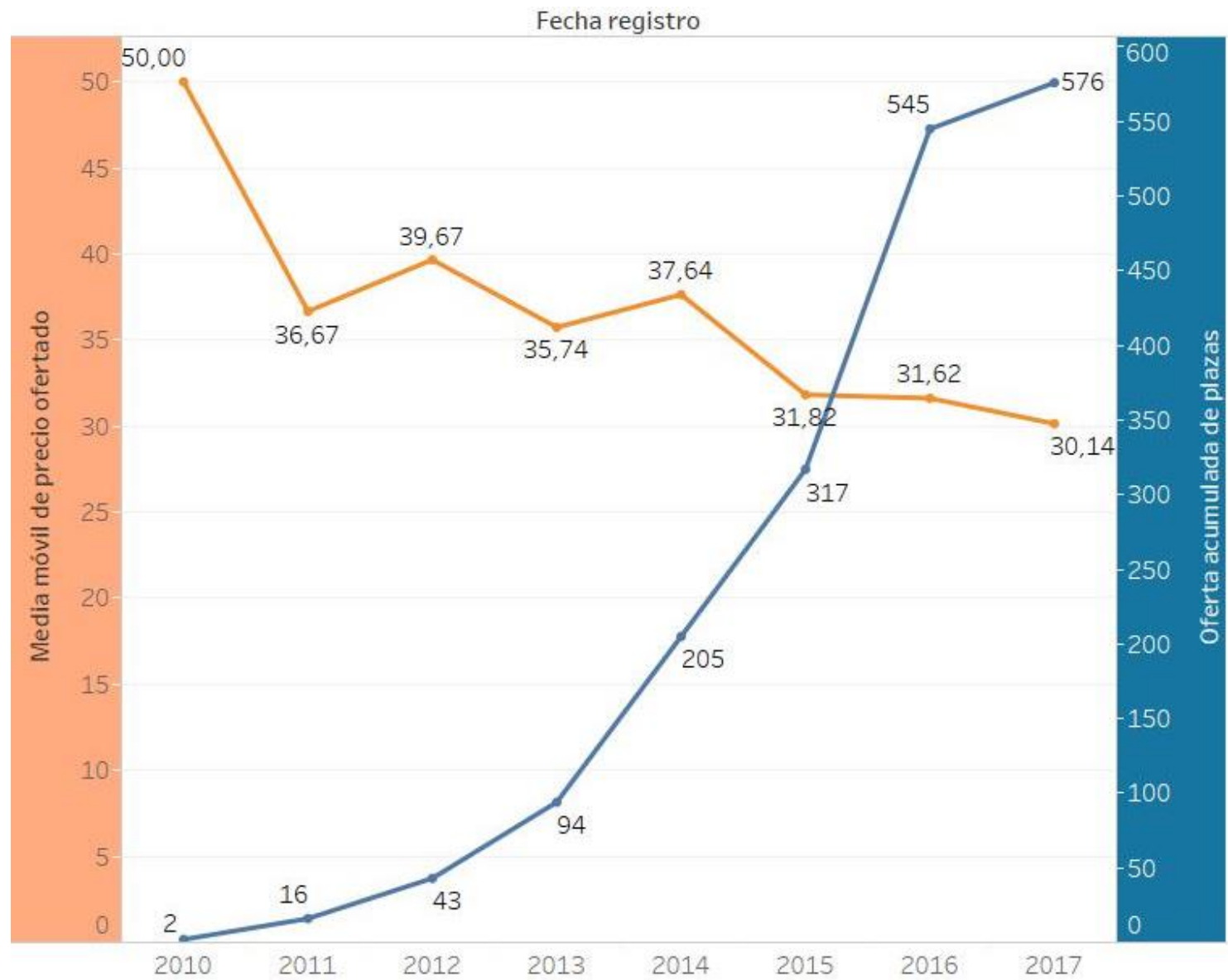


El gráfico 2 muestra la reducción de las tarifas promedios ofertadas en la página web de Airbnb, frente al incremento histórico en la oferta de hospedaje, es decir, el precio está condicionado por la competencia o, en su defecto, por Airbnb, ya que de acuerdo a sus datos históricos sabe cómo evolucionará la demanda.

\section{Gráfico 3}

Tendencia de rendimiento de Airbnb en Cuenca

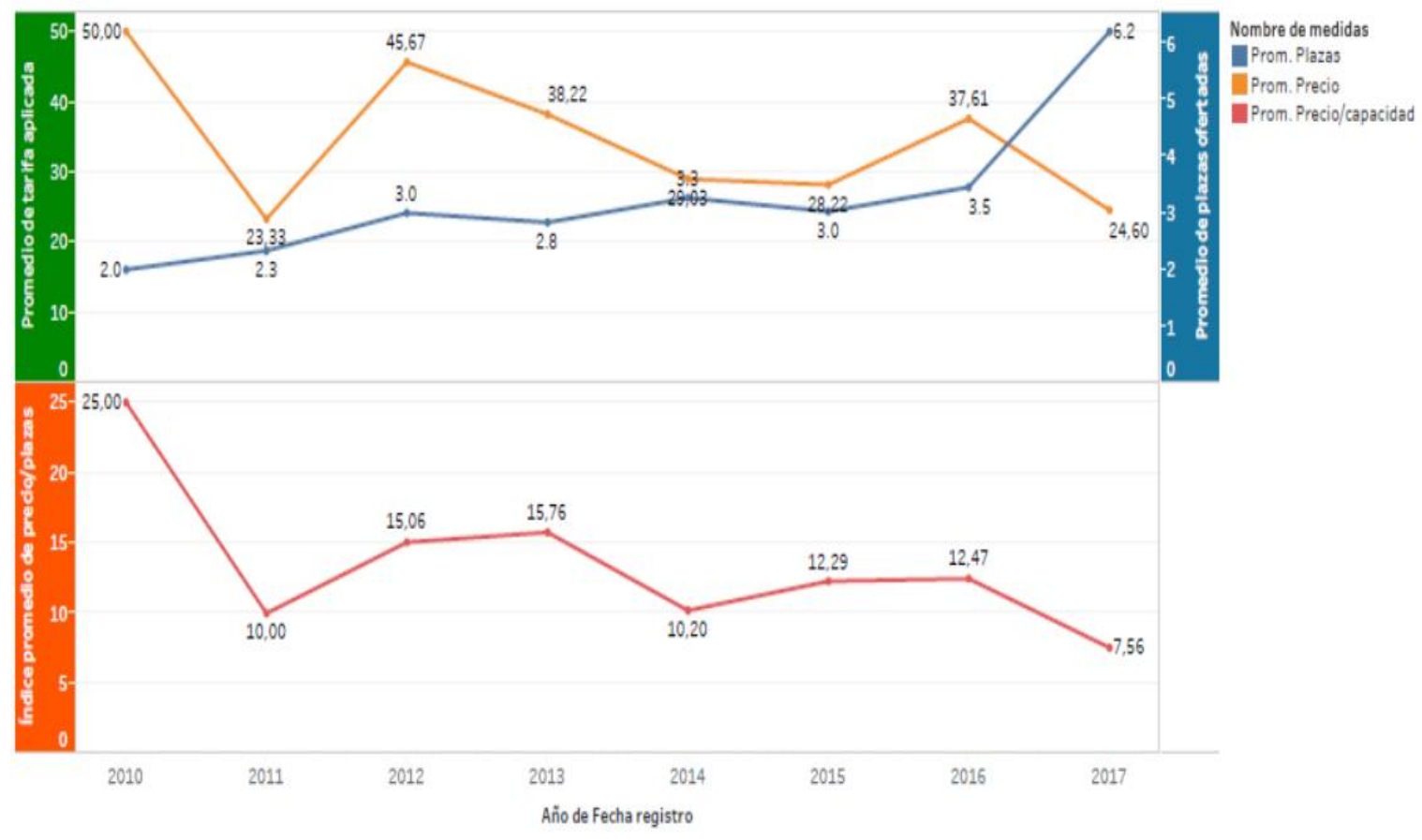

El gráfico 3 muestra la tendencia de rendimiento de Airbnb en Cuenca; el promedio de plazas y precio estima el movimiento de la tarifa en el tiempo. La tarifa promedio en el tiempo dependerá de la mayor o menor oferta de locales de este tipo. Al parecer, la tarifa de este tipo de establecimientos reacciona perfectamente a la demanda de alojamiento.

Desde el año 2010, las plazas promedio de los locales aperturados crece desde 2 hasta 6.2 plazas promedio; en cambio, la tarifa va decreciendo en el tiempo desde $\$ 50$ hasta $\$ 24.60$ por persona, en el año 2017 . El indicador precio/plaza tiene una reacción decreciente provocada por el incremento del número de plazas ofertadas, pero también por una reducción de la tarifa promedio. Los 


\section{Freddy Edgar Espinoza Figueroa, Segundo Amador Freire Chaglla}

Características y evolución de la oferta de alojamiento de Airbnb en una

ciudad patrimonio cultural de la humanidad. Caso Cuenca (Ecuador)

negocios de Airbnb, cuyo punto de equilibrio no se ha podido determinar para relacionarlo con el de establecimientos regulados, reducen su tasa de ganancia, desde el año 2010 con un índice de $\$ 25$ (2010) a una de \$7.56 (2017), por persona. Esto significa que el negocio de hospedaje no regulado pronto dejaría de tener ganancias afectando directamente a los establecimientos ya instalados, a cambio de una tasa de pérdidas importante.

\section{Gráfico 4}

Promedio de evaluaciones (comentarios) de usuarios a los servicios Airbnb

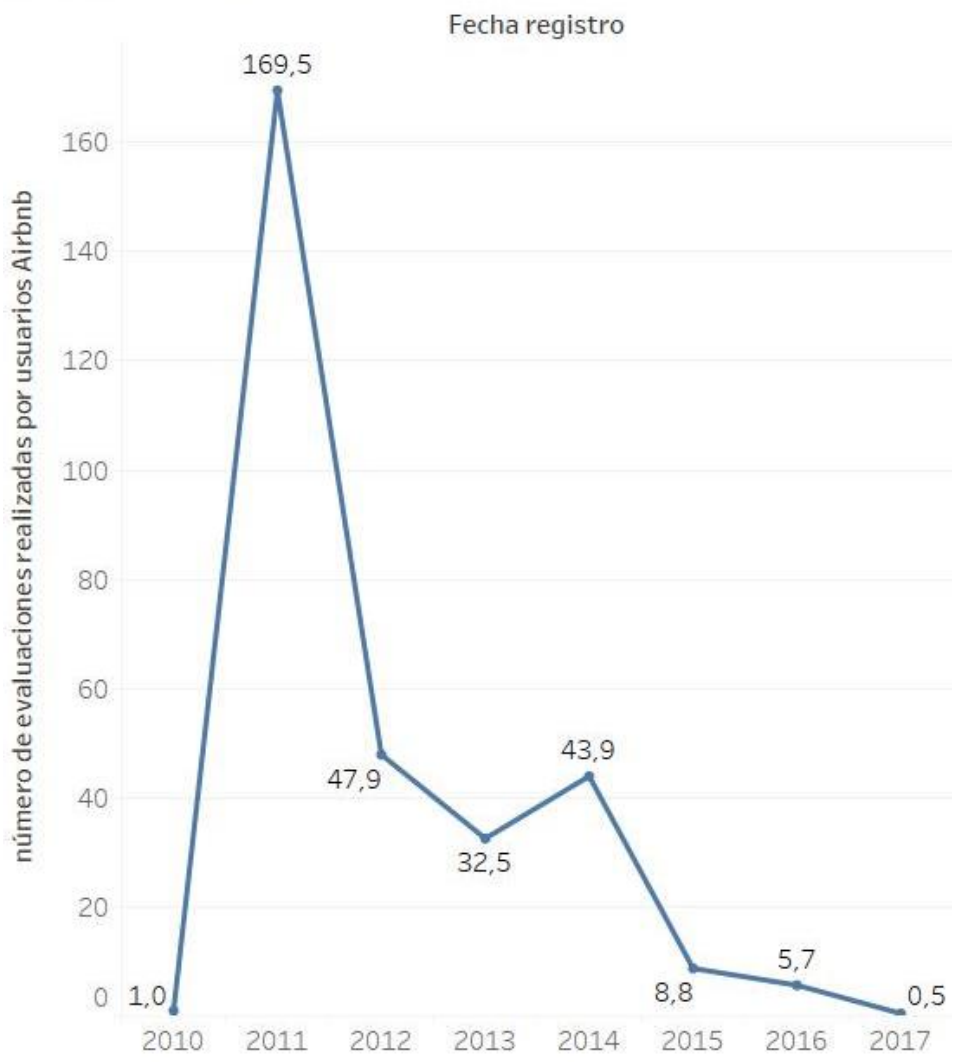

El número de evaluaciones (comentarios) recibidos por parte de los usuarios (huéspedes) se reduce con el pasar del tiempo, considerando que después de usar los servicios, el anfitrión solicita dejar un comentario que sirva de referencia para futuros clientes. Esta tendencia pudiera tener una explicación en la reducción de usuarios por tanto de la demanda. 


\section{Discusión y conclusiones}

Es preciso mencionar que la economía colaborativa se intenta posicionar como un empoderamiento del individuo, no obstante, parece ser un disfraz de la

economía capitalista de mercado, según un informe de ESADE, el cual menciona que el $95 \%$ de dicha actividad se queda en el $1 \%$ de las plataformas, el $50 \%$ de transacciones la tienen 17 empresas valoradas en miles de millones de dólares (Lorenzo, 2016), esas cifras muestran el poder dominante de grandes corporaciones (Bulchand Gidumal \& Melián Gonzáles, 2016), característica intrínseca del capitalismo, entonces: ¿Es una innovación disruptiva para el cambio social? (Christensen, Baumann, Ruggles, \& Sadtler, 2006).

La irrupción de la llamada economía colaborativa, específicamente en alojamiento a través de plataformas de intermediación web, “(...)oferta servicios propios de una economía turística(...)" (Cabrerizo, Sequera, \& Bachiller, 2016, p. 79), sin embargo, precarizan el empleo inexistente, mal remunerado o con alta temporalidad, dando lugar a que los destinos enfrenten grandes desafíos (Cabrerizo et al., 2016), tal es el caso del distrito Centro de Madrid con 132.644 habitantes y una superficie aproximada de 522 hectáreas, al 1 de enero de 2016 existían 215 plazas por cada 1.000 habitantes, a pesar de que TURESPAÑA respecto a esa relación sostiene que lo adecuado -en cuanto a plazas reguladases 204 por cada 1.000 habitantes (Cabrerizo et al., 2016). Inside Airbnb, proporciona datos de esta oferta a nivel de barrio, "...7.746 ofertas de Airbnb en Madrid, el $56,1 \%$ se localizan en el distrito Centro (4.177). Por barrios, Embajadores concentra un mayor número (1.107), seguido de Universidad (906), Palacio (669), Sol (544), Justicia (501) y Cortes (450)" (Cabrerizo et al., 2016, p. 81). Aparte de las preocupaciones concernientes a la eventual pérdida de valores del patrimonio cultural, existen otras como el impacto de Airbnb en el mercado inmobiliario, San Francisco, Nueva York, Vancouver y Berlín han empezado a preocuparse por aquello, pues temen que Airbnb reduzca la oferta de alojamiento regulado a largo plazo, esto implicaría una elevación de los costos en los destinos, además, a nivel urbano la población residente migraría a sitios que tradicionalmente no estaban destinados para ser viviendas o en su defecto que dicha población no tenga donde vivir (Neeser, 2015). 
Airbnb no es la única plataforma en Cuenca (Tripadvisor, Booking, Backpackers) no obstante, constituye una forma flexible de generar ingresos, es más fácil emprender un "negocio" de alquiler de habitaciones en una zona de la ciudad consolidada -con variedad de servicios y atractivos- tal es el caso del Centro Histórico de Cuenca (Gráfico 1), lo cual puede resultar positivo a corto plazo, considerando que el mercado se diversifica, pero a largo plazo, varios segmentos de mercado de alojamiento tradicional pueden migrar hacia la oferta más económica o, en el peor de los casos, cerrar sus instalaciones. En cualquiera de los dos escenarios, pierde el patrimonio cultural, puesto que los centros históricos son espacios sensibles que no están preparados para esta situación.

Dentro de las consecuencias se podría enumerar: i) el incremento de precios inmobiliarios; ii) la presión urbanística desmedida; iii) el desbordamiento de los servicios públicos; iv) la pérdida de valores de edificaciones patrimoniales al adecuar sitios de hospedaje en espacios no destinados para aquello; iv) la migración residencial a espacios que habitualmente no estaban destinados para la vivienda, o en su defecto $v$ ) el des proveimiento del acceso a la vivienda por el incremento de las tarifas de arriendo; aspectos que pueden dar paso a una incipiente gentrificación o los como el caso de la parroquia de San Joaquín caracterizada por actividades agrícolas y ahora por la construcción de megaurbanizaciones (Deustche \& Gendel Ryan, 2015), generando un escenario al que se denominará: "la tragedia de los comunes" (Dans, 2010).

La apertura de este tipo de negocios ha sido constante durante los últimos años, sin embargo, existe un descenso en la tarifa promedio a lo largo del tiempo (Tabla 2), considerando que la fijación de precios es orientada por Airbnb, tal vez este comportamiento no este condicionado solamente por la competencia, sino por el escenario actual de la ciudad de Cuenca, carente de un modelo de gestión que asuma integralmente las competencias traspasadas en 2001, además de otros problemas ya mencionados (aeropuerto y Tranvía), generando una suma de situaciones que perjudican las ganancias de este tipo de negocios, eventualmente pronto dejarían de percibirlas (Tabla 3).

A esto se adiciona el decrecimiento de usuarios en relación al promedio de evaluaciones (comentarios) (Tabla 4), por lo que es necesario entender que gestionar el turismo es gestionar la complejidad, el espacio intermedio entre política y negocio (Capece, 2012), sin perder de vista los elementos que hacen posible esta actividad: el patrimonio cultural y las relaciones que se generan entre la comunidad anfitriona y los turistas (ICOMOS, 1999). 
Freddy Edgar Espinoza Figueroa, Segundo Amador Freire Chaglla

Características y evolución de la oferta de alojamiento de Airbnb en una

ciudad patrimonio cultural de la humanidad. Caso Cuenca (Ecuador)

Por otro lado, es difícil comprender y asimilar las interrelaciones formadas entre el sector público y privado, las propuestas de cada sector se apoyan en visiones sesgadas que obedecen a la carencia de datos estadísticos de la realidad, generando "fracasos premeditados", pues se requiere del turismo algo que no puede dar (Capece, 2012).

La recesión en torno al alojamiento turístico es para establecimientos regulados y no regulados, no obstante, la capacidad de innovación es una constante para los últimos (característica que debe ser imitada por los regulados), de

existir problemas, pronto innovaran en cuanto a espacio, características del servicio, servicios complementarios, entre otros aspectos, con el fin de ser más competitivos entre ellos y con el alojamiento regulado que tiene gastos más altos, y está obligado a tributar y cumplir con la normativa. Actualmente, el sector privado pide regulación para los informales -por así decirlo- pero una vez regulados cual sería el escenario: ¿Más competidores? Sí, más alojamientos "regulados" de todo tipo, de preferencia en el Centro Histórico, sin estudiar la demanda en una clara tendencia de sobreoferta, mucho menos los efectos sobre el patrimonio cultural.

Finalmente, desde la perspectiva del empresario de alojamiento regulado, este fenómeno quizá sea "lo peor que ha podido pasar para el turismo", no obstante, desde la perspectiva del usuario/demandante es posiblemente "lo mejor que ha pasado", en virtud de lo fácil que resulta acceder a bienes y servicios (Owyang et al., 2013); desde la mirada de la persona que oferta un bien o servicio de este tipo, probablemente represente una oportunidad laboral que genera ingresos extras en respuesta a una recesión económica.

Para una ciudad patrimonial que pretende consolidarse como un destino turístico, la sobreoferta existente en alojamiento representa un grave problema, puesto que la autenticidad, integridad y valores patrimoniales del sitio empiezan a menoscabarse paulatinamente en aras del "desarrollo". Regulación, gestión, investigación, innovación, planificación, control y regulación en la ciudad patrimonial son acciones inaplazables e ineludibles.

Es inexorable recurrir a la investigación con rigurosidad científica en torno al tema, sin olvidar que las limitaciones para estudiar el fenómeno están condicionadas por el costo de reserva "para saber más a fondo el comportamiento de Airbnb". Es momento de relegar la ilusoria idea de que este fenómeno está relacionado solamente con el turismo, específicamente con el alojamiento, puesto 
que, poco a poco, diferentes estudios dejan entrever los efectos sobre la población, el patrimonio cultural, la planificación urbana, la economía, entre otros aspectos.

\section{Bibliografía}

Ascanio Guevara, A. (2012). Estadística del turismo (Ediciones). Bogotá.

Astudillo, G. (2016, febrero 16). Operativos contra hoteles y restaurantes informales se inician en Cuenca. El Comercio. Cuenca. Recuperado de: http://www.elcomercio.com/actualidad/operativos-hoteles-restaurantesinformales-cuenca.html

Bostman, R., \& Rogers, R. (2011). What's mine is yours: how collaborative consumption is changing the way we live (Collins). London.

Bulchand Gidumal, J., \& Melián Gonzáles, S. (2016). Una guía para entender la economía colaborativa (Imecola.co). Las Palmas.

Cabrerizo, C., Sequera, J., \& Bachiller, P. G. (2016). Entre la turistificación y los espacios de resistencia en el centro de Madrid: Algunas claves para (re)pensar la ciudad turística. Ecología Política, (52), 78-83.

Capece, G. (2012). Turismo: Gestión de la complejidad (Fundación). Buenos Aires.

Caraballo Perichi, C. (2011). Valores patrimoniales. Hacia un manejo integral y participativo. In Patrimonio Cultural un enfoque diverso y comprometido (UNESCO). México D.F.

Christensen, C. M., Baumann, H., Ruggles, R., \& Sadtler, T. M. (2006). Innovacion disruptiva para el cambio social. Harvard Business Review, 84(12), 58-65. Retrieved from http://dialnet.unirioja.es/servlet/articulo?codigo=2190622\&info=resumen\&idi oma $=$ SPA

Cioce-Sampaio, C., \& Silva-Segovia, Y. (2015). Turismo Como Fenomeno Humano: Princios Para Pensar En La Ecosocioeconomia. TURydDES, 8(19), 1-11.

Coraggio, J. L. (2011). Economía social y solidaria. El trabajo antes que el capital. Quito. Retrieved from http://www.collaboriamo.org/media/2014/04/collabecon-draft16130531132802-phpapp02-2.pdf

Dans, E. (2010). Todo va a cambiar (Deusto).

Departamento de Investigación Facultad de Ciencias de la Hospitalidad. (2016). Turismo en la Ciudad de Cuenca. Cuenca. 
Deustche, R., \& Gendel Ryan, C. (2015). El bello arte de la gentrificación. In Observatorio Metropolitano de Madrid (Ed.), El mercado contra la ciudad (Traficante, pp. 27-52). Madrid.

El Mercurio. (2014, agosto 6). La hotelería informal gana espacio. El Mercurio. Cuenca. Recuperado de: http://www.elmercurio.com.ec/442649-ganaespacio-hoteleria-informal-en-cuenca/

El Tiempo. (2016, noviembre 14). El alojamiento informal es una realidad latente. El Tiempo. Cuenca. Recuperado de: http://www.eltiempo.com.ec/noticias/cuenca/2/402375/el-alojamientoinformal-es-una-realidad-latente

Evans, D., \& Schmalensee, R. (2016). The Matchmakers: The new economics of multisided platforms (Harvard $\mathrm{Bu}$ ). Boston.

Expreso. (2016, junio 5). La Asociación Hotelera de Cuenca se declara en crisis. Expreso. Cuenca. Recuperado de: http://www.expreso.ec/actualidad/laasociacion-hotelera-de-cuenca-se-declara-en-crisis-AB388534

Felber, C. (2012). La economía del bien común: un modelo económico que supera la dicotomía entre capitalismo y comunismo para maximizar el bienestar de nuestra sociedad. (Grupo Plan). Barcelona.

Fernández de Paz, E. (2006). De tesoro ilustrado a recurso turístico: el cambiante significado del patrimonio cultural. Pasos Revista de Turismo Y Patrimonio Cultural, 4(1). $\quad$ Retrieved from http://www.pasosonline.org/Publicados/4106/PASOS07.pdf\#page $=12$

Gallagher, L. (2017). The Airbnb story (Houghton M). New York.

García Álvarez, M., Osorio Guerrero, P., \& Pastor Herrera, L. (2017). Estudio sobre los impactos socio-económicos en Cuenca de la migración residencial de norteamericanos y europeos: aportes para una convivencia armónica local. Cuenca.

Gonzáles, J. (2017, septiembre 23). Uber no podrá funcionar en Guayaquil. El Comercio.

Quito.

Recuperado

de:

http://www.elcomercio.com/actualidad/uber-funcionamiento-guayaquilecuador-taxis.html

ICOMOS. Carta internacional sobre el turismo cultural. La Gestión del Turismo en los sitios con Patrimonio (1999). México. Retrieved from http://www.icomos.org/charters/tourism_sp.pdf

Ilustre Concejo Cantonal. Estatuto de la Fundación Municipal "Turismo para Cuenca" (2005).

Ecuador. Retrieved from http://www.cuenca.gob.ec/?q=system/files/207_ESTATUTO DE LA 
FUNDACION MUNICIPAL.doc

Ilustre Consejo Municipal de Cuenca. Ordenanza para la gestión y conservación de las áreas históricas y patrimoniales del cantón Cuenca (2010). Ecuador.

Retrieved from http://www.cuenca.gov.ec/?q=node/8993

INEC. (2015). Población superficie (Km2), densidad poblacional a nivel parroquial. Retrieved from http://www.inec.gob.ec/tabulados_CPV/2_Densidad_Pobla_Nac_Prov_Cant Parr.XLSX a

Kostakis, V., \& Bauwens, M. (2014). Network society and future scenarios for a collaborative economy (Springer).

López, J. (2016, marzo 9). Usuarios de Airbnb en México crecieron 188\%. El Financiero. México D.F. Recuperado de: http://www.elfinanciero.com.mx/empresas/usuarios-de-airbnb-en-mexicocrecieron-188.html

Lorenzo, M. (2016, octubre). La mitad de la economía colaborativa está en manos de 17 empresas. Tendencias 21. Recuperado de: http://www.tendencias21.net/La-mitad-de-la-economia-colaborativa-esta-enmanos-de-17-empresas_a43293.html

Martin, C. J. (2016). The sharing economy: A pathway to sustainability or a nightmarish form of neoliberal capitalism? Ecological Economics, 121, 149159. https://doi.org/10.1016/j.ecolecon.2015.11.027

Ministerio de Turismo. Reglamento de alojamiento turístico (2015). Ecuador. Retrieved from http://www.turismo.gob.ec/wp-content/uploads/2016/07/1802-2016-Reformas-al-Reglamento-de-Alojamiento.pdf

Ministerio de Turismo. (2017). Anuario de Estadísticas Turísticas. Retrieved from Ministerio de Turismo

Neeser, D. (2015). Does Airbnb Hurt Hotel Business: Evidence from the Nordic Countries. Universidad Carlos III de Madrid. https://doi.org/10.13140/RG.2.1.4939.8248

Organización Mundial de Turismo (OMT). (2010). Recomendaciones internacionales para estadísticas de turismo 2008. Madrid/New York.

Owyang, J., Tran, C., \& Silva, C. (2013). The Collaborative Economy: Products, services, and market relationships have changed as sharing startups impact business models. To avoide disruption, companies must adopt the Collaborative Economy Value Chain. Altimeter, 1-27. Retrieved from http://www.collaboriamo.org/media/2014/04/collabecon-draft16-

130531132802-phpapp02-2.pdf 
ciudad patrimonio cultural de la humanidad. Caso Cuenca (Ecuador)

Pascual, G. D. (2015). La Regulación de la Economía Colaborativa. Revista CEFLEGAL, 175-176, 61-104. Retrieved from http://roderic.uv.es/bitstream/handle/10550/54302/112244.pdf?sequence=1

Pizarro, E. (2017, febrero 15). Airbnb avanza como opción de alojamiento en la Argentina. La Nación. Buenos Aires. Recuperado de: http://www.lanacion.com.ar/1984821-airbnb-avanza-como-opcion-dealojamiento-en-la-argentina

Rojano Uscanga, A. (2016, diciembre 23). Francia aprueba la tasa fiscal "Google." El Dictamen. México D.F. Recuperado de: http://eldictamen.mx/2016/12/columnas/ahora-y-aqui/francia-aprueba-latasa-fiscal-google/

Smith, N., \& Williams, P. (2013). Gentrification of the city (Routledge).

UNESCO. (2017). Centro histórico de Santa Ana de los Ríos de Cuenca. Retrieved January 1, 2017, from http://whc.unesco.org/es/list/863\#top

Vaughan, R., \& Daverio, R. (2016). Assesing the size and presence of the collaborative economy in Europe. Recuperado de: http://ec.europa.eu/DocsRoom/doucuments/16952/attachments/1/translation s/en/renditions/pdf 

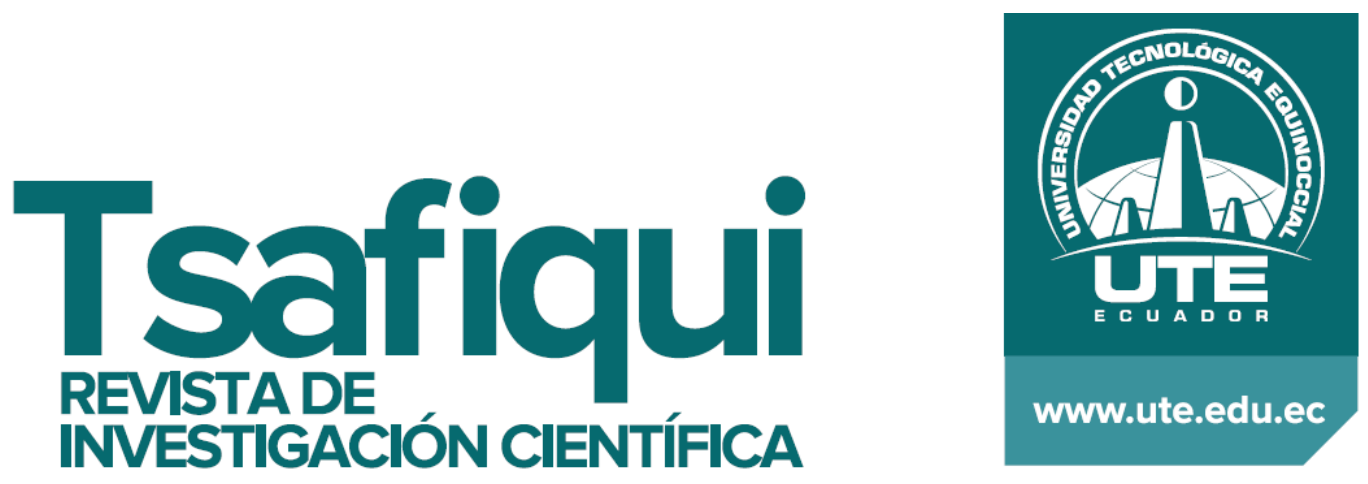